\title{
Synthetic Lethality Is a Novel and Potential Paradigm for Precision Medicine in Advanced Hepatocellular Carcinoma
}

\author{
Mingyu Chen ${ }^{a, b} \quad$ Xiujun Cai ${ }^{a, b}$ \\ aDepartment of General Surgery, Sir Run-Run Shaw Hospital, Zhejiang University, \\ Hangzhou, China; ${ }^{b}$ Key Laboratory of Endoscopic Technique Research of Zhejiang Province, \\ Sir Run-Run Shaw Hospital, Zhejiang University, Hangzhou, China
}

Dear Editor,

We read with great interest the article by Bouattour et al. [1] about the systemic treatment for advanced hepatocellular carcinoma (HCC). This study showed a practical overview of current and future systemic treatment options for advanced HCC. However, for precision medicine in HCC, only traditional targeted therapies have been described. In fact, synthetic lethal treatment is also becoming a potential paradigm for cancer treatment in HCC.

Synthetic lethality describes the situation in a cell or organism whereby individual gene mutations have little or no effect, but the co-occurrence of mutation in both genes results in death. Synthetic lethal treatment can facilitate the indirect targeting of cancer mutation (lack of targeted drug) through the identification of a second-site synthetic lethal target, which can range from oncogenes to tumor suppressors, and even genetic background. Research over the past decade revealed that synthetic lethality was one of the most effective treatments for advanced HCC. For example, Wang et al. [2] indicated that a synthetic lethal drug combination of sorafenib and MEK inhibition (selumetinib) was most likely to be effective in liver cancer with high basal phospho-ERK levels. Zhang et al. [3] showed that combination therapy with both poly(ADP-ribose) polymerases (PARPs) inhibition and histone deacetylases inhibition might be a strategy for the therapy of sensitive HCC cells. Moreover, an objective and effective response has been observed in synthetic lethal therapy for patients with $B R C A 2$ germline truncation mutations to liver cancer using PARP inhibition (olaparib) in a clinical trial (NCT02715089). Furthermore, some common gene mutations in liver cancer show potential and promising targets and opportunities for cancer treatment using synthetic lethal interaction. For example, ATR inhibitors in ARID1A-deficient cells results in cell death [4], inhibition of APE1 promotes apoptosis in PTEN-deficient cells, and PI5P4K kinases are essential for cell growth in the absence of p53 [5]. Therefore, synthetic lethality, which can expand the repertoire of anticancer therapeutic targets and have the potential to drive more drug discovery, maybe plays an important role in the management and treatment of liver cancer.

Despite its potential, synthetic lethality still faces some limits and challenges. For example, HCC generally emerges from the liver with deteriorated function, so synthetic lethal treatment may give rise to severe hepatotoxicity that may limit the application of combination therapies in the clinical setting. Besides, therapy

Xiujun Cai

Department of General Surgery, Sir Run-Run Shaw Hospital, Zhejiang University

No. 3 East Qingchun Road

Hangzhou 310016 (China)

srrsh_cxj@zju.edu.cn 
resistance is a potential challenge. Although recent research revealed that therapy resistance commonly resulted from conditional synthetic lethality, to find the targeted genes or key pathways still needs a long way to go.

Nevertheless, synthetic lethality may become one of the promising directions for precision medicine in the treatment for liver cancer.

\section{Disclosure Statement}

The authors have no conflicts of interest to declare.

\section{Funding Sources}

The authors did not receive any funding.

\section{Author Contributions}

Mingyu Chen and Xiujun Cai wrote the paper, and both authors read and approved the final manuscript.

\section{References}

1 Bouattour M, Mehta N, He AR, Cohen EI, Nault JC. Systemic Treatment for Advanced Hepatocellular Carcinoma. Liver Cancer. 2019;8(5):341-58.

2 Wang C, Jin H, Gao D, Lieftink C, Evers B, Jin G, et al. Phospho-ERK is a biomarker of response to a synthetic lethal drug combination of sorafenib and MEK inhibition in liver cancer. J Hepatol. 2018 Nov;69(5):1057-65.

3 Zhang JX, Li DQ, He AR, Motwani M, Vasiliou V, Eswaran J, et al. Synergistic inhibition of hepatocellular carcinoma growth by cotargeting chromatin modifying enzymes and poly (ADP-ribose) polymerases. Hepatology. 2012 Jun;55(6):1840-51.

4 Williamson CT, Miller R, Pemberton HN, Jones SE, Campbell J, Konde A, et al. ATR inhibitors as a synthetic lethal therapy for tumours deficient in ARID1A. Nat Commun. 2016 Dec;7(1):13837.

5 Lokody I. Signalling: a new target for p53-null tumours. Nat Rev Cancer. 2014 Jan;14(1):8-9. 